\title{
Synthesis of various isoniazidothiazolidinones and their imidoxy derivatives of potential biological interest
}

\author{
Ranjana Sharma, Devendra P. Nagda, and Ganpat L. Talesara* \\ Synthetic Organic Chemistry Laboratory, Department of Chemistry, M. L. Sukhadia University \\ Udaipur (Raj.)- 313 001, India \\ E-mail: gtalesara@yahoo.com; rdchemorg@yahoo.co.in
}

\begin{abstract}
A variety of phthalimido[2-aryl-3-(5'-(4''-pyridyl)-1',3',4'-thiadiazol-2'-yl)-4-oxothiazolidin-5yl] ethanoates 7a-h and 3-N-alkoxyphthalimido-2-isonicotinoylhydrazido-1,3-thiazolidin-4-ones 9a-c were synthesized using thiosemicarbazide of isoniazid 2 by two alternative pathways. The structures of these compounds were confirmed by IR, NMR $\left({ }^{1} \mathrm{H} \&{ }^{13} \mathrm{C}\right)$ and Mass spectral studies. Synthesized compounds 7a-h and 9a-c were evaluated for their antimicrobial activity. Some of the compounds exhibited good antimicrobial activity compared to standard drugs. The structure activity relationships of synthesized compounds have also been studied in order to develop the most potential antimicrobial agent for preclinical evaluation.
\end{abstract}

Keywords: Isoniazid, thiadiazole, thiazolidinones, imidoxy, antimicrobial studies

\section{Introduction}

Tuberculosis is believed to be present in about one third of the world's population. ${ }^{1}$ The increasing incidence of multi-drug-resistant tuberculosis is emerging as a major infectious disease problem throughout the world. ${ }^{2}$ Most of the drug resistant clinical isolates of the tubercle bacillus are resistant to isoniazid which is a first line antituberculous drug. ${ }^{3}$ This antibiotic was shown to act on Mycobacterium tuberculosis by inhibiting a 2-trans-enoyl-acyl carrier, protein reductase, called InhA. ${ }^{4}$ To this day, isoniazid is better known for its more commonly observed effects on vitamin $B_{6}$ and the resultant peripheral neuropathy that can occur in patients who don't receive adequate amounts of vitamin $\mathrm{B}_{6} \cdot{ }^{5-7}$ Despite the large number of compounds containing the isoniazid moiety which have already been synthesized and tested, there is still a need for new compounds of this kind $^{8,9}$ due to the increasing resistance of bacterial strains to certain type of antibiotics. ${ }^{10}$ Furthermore, the structure and chemistry of the 1,2,3-thiadiazole system has been actively investigated the last few years. ${ }^{11-13}$ Its derivatives are useful in the treatment of hyperproliferative disorders including tumor growth, angiogenesis and lumphoproliferative 
symptoms. ${ }^{14}$ Moreover, derivatives of this system are applied in treatment and/or prevention of morbid states mediated by oxytocin including premature labour and dysmenorhea. ${ }^{15}$ The $1,2,3-$ thiadiazole moiety is crucial for the antibacterial activity of new carbapenems ${ }^{16}$ as well as for the efficacy of some pesticides. ${ }^{17}$

4- Thiazolidinone derivatives have also been prepared and used as intermediate in organic synthesis. ${ }^{18}$ Some biological activities such as bactericidal, pesticidal, fungicidal, insecticidal, anticonvulsant, tuberculostatic, anti-inflammatory, antithyrodial, potentiation of pentobarbital induced sleeping time are associated with 4-thiazolidinone derivatives. ${ }^{19-21}$ The imidoxy compounds have been tested and evaluated mainly for antiepileptic and anticancer activities. ${ }^{22}$ The most promising imidoxy anticonvulsant was ethyl phthalimidoxy acetate ${ }^{23}$ and they may play an important role in the peptide synthesis and inhibition of human leukocyte elastase. ${ }^{24}$

In this paper, we describe the synthesis of two series of isonicotinic acid hydrazide (INH, isonazid) derivatives, phthalimido[2-aryl-3-(5'-(4'-pyridyl)-1', $3^{\prime}, 4^{\prime}$-thiadiazol-2'-yl)-4oxothiazolidine-5-yl]ethanoates 7a-h and 3-N-alkoxyphthalimido-2-isonicotinoylhydrazido-1,3thiazolidin-4-ones 9a-c and the results of their testing for antibacterial and antifungal activity. The influence of structural modification on biological activity is also discussed.

\section{Results and Discussion}

In the present work isonicotinic acid hydrazide (INH) 1 was converted into 2-[aryl]-3-(5'-(4'pyridyl)-1',3',4'-thiadiazol-2'-yl)-4-oxo-thiazolidin-5-yl ethanoic acid $\mathbf{5 a - h}$ by its reaction with ammonium thiocyanate in acidic medium to synthesize isonicotinoylthiosemicarbazide 2 which gave 2-amino-5-(4'-pyridyl)-1,3,4-thiadiazole 3 on cyclization in presence of conc. sulfuric acid. When $\mathbf{3}$ was refluxed with various aldehydes, it gave the corresponding arylidene derivatives 4a$\mathbf{h}$ which on treatment with mercaptosuccinic acid furnished thiazolidinone derivatives $\mathbf{5 a - h}$. In order to synthesize imidoxy derivative of isoniazidothiazolidinone compounds $\mathbf{5 a - h}$ were treated with thionyl chloride in toluene to synthesize its acid chloride derivatives 6a-h and finally phthalimido[2-aryl-3-(5'-(4"'-pyridyl)-1',3',4'-thiadiazol-2'-yl)-4-oxothiazolidin-5-yl] ethanoates 7a-h were synthesized by the reaction of N-hydroxyphthalimide with $\mathbf{6 a - h}$.

In parallel, isonicotinoylthiosemicarbazide $\mathbf{2}$ on cyclization with chloroacetic acid in the presence of sodium acetate in dry ethanol yielded 2-isonicotinoylhydrazido-1,3-thiazolidinone 8. $\omega$-Bromoalkoxyphthalimide were condensed with $\mathbf{8}$ in alcohol using pyridine as base furnishing 3-N-alkoxyphthalimido-2-isonicotinoylhydrazido-1,3-thiazolidin-4-ones 9a-c. Various organic bases were used to eliminate $\mathrm{HBr}$ such as pyridine, piperidine and triethylamine. The reactions gave poor yields when triethylamine was used and a longer reflux time was necessary. Piperidine as a base generally gave a sticky product which could not be crystallized but when the reaction was carried out with two moles of pyridine, better results were obtained. The structures of all synthesized compounds were in agreement with their spectral and analytical data. 


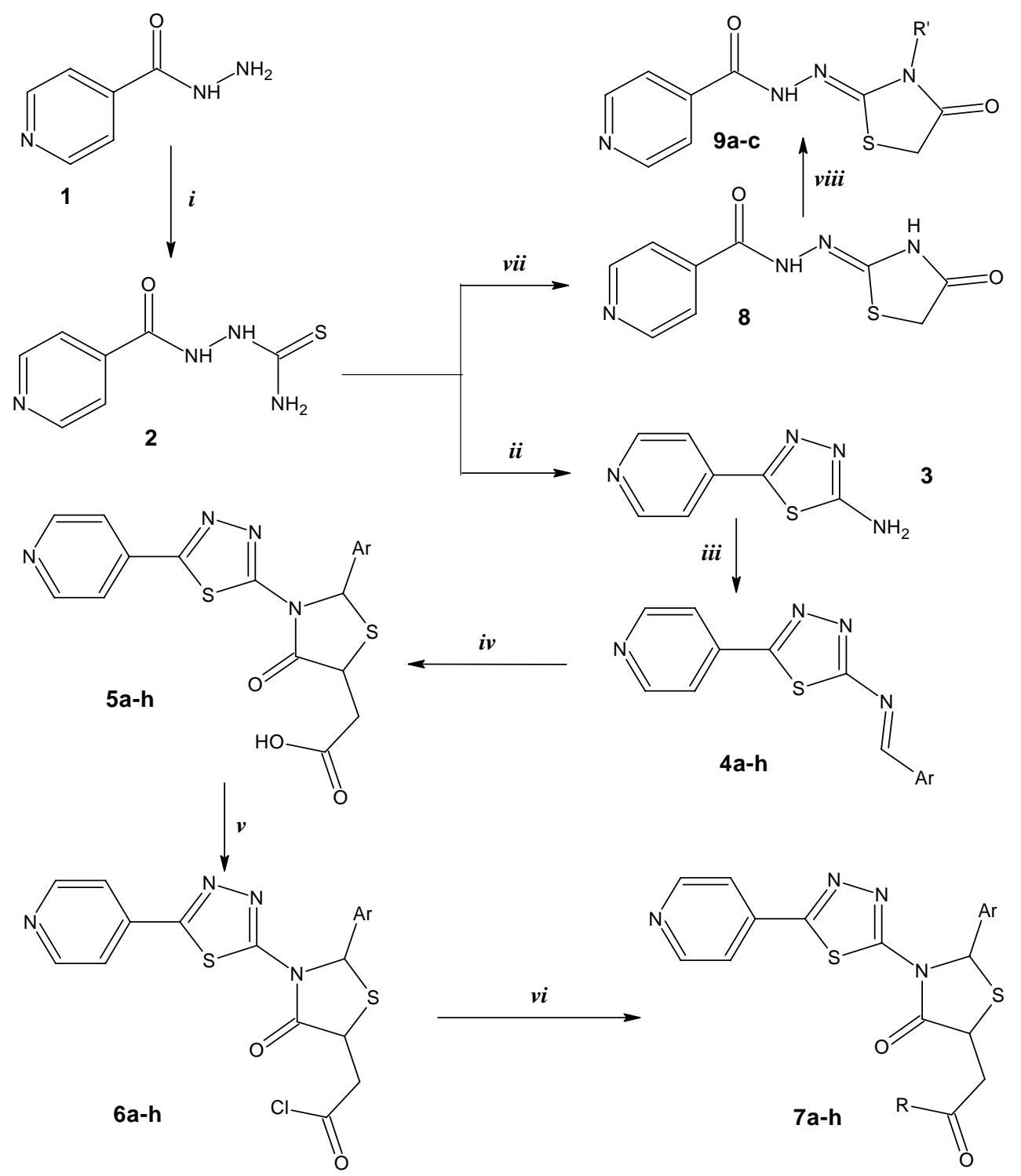

Scheme 1. $\operatorname{Ar}(\mathbf{a}-\mathbf{h})=4-\mathrm{OCH}_{3} \cdot \mathrm{C}_{6} \mathrm{H}_{4}, 4-\mathrm{Cl}_{6} \mathrm{C}_{6} \mathrm{H}_{4}, 3,4,5-\mathrm{OCH}_{3} \cdot \mathrm{C}_{6} \mathrm{H}_{2}, 3-\mathrm{NO}_{2} \cdot \mathrm{C}_{6} \mathrm{H}_{4}, 4-\mathrm{NO}_{2} \cdot \mathrm{C}_{6} \mathrm{H}_{4}, 4-$ $\left(\mathrm{CH}_{3}\right)_{2} \mathrm{NH} . \mathrm{C}_{6} \mathrm{H}_{4}, \mathrm{C}_{6} \mathrm{H}_{5}, \mathrm{C}_{4} \mathrm{H}_{3} \mathrm{O}$ (2-furyl) $\mathbf{R}=$ phthalimidoxy, $\mathbf{R}$ ' = phthalimidoxy alkyl. Reagents and reaction conditions: (i) $\mathrm{NH}_{4} \mathrm{SCN}, 1 \mathrm{~N} \mathrm{HCl}$; (ii) Conc. $\mathrm{H}_{2} \mathrm{SO}_{4}, \mathrm{NH}_{3}$; (iii) ArCHO, EtOH; (iv) $\mathrm{ZnCl}_{2}$, 2-mercaptosuccinic acid; (v) $\mathrm{SOCl}_{2}$, Toluene; (vi) DMF, TEA, N-hydroxyphthalimide; (vii) $\mathrm{ClCH}_{2} \mathrm{COOH}$, AcONa, EtOH; (viii) Pyridine, EtOH, phthalimidoxyalkylbromide

\section{Antimicrobial Screening}

In the present investigation, various substituted phthalimido[2-aryl-3-(5'-(4'-pyridyl)-1',3',4'thiadiazol-2'-yl)-4-oxothiazolidin-5-yl]ethanoates $\mathbf{7 a - h}$ and 3-N-alkoxyphthalimido-2isonicotinoylhydrazido-1,3-thiazolidin-4-ones 9a-c derivatives have been evaluated for their antimicrobial activity. 
Table 1. Antimicrobial activity of the synthesized compounds 7a-h \& 9a-c. Zone of inhibition $(\mathrm{mm})$ (activity index) ${ }^{\text {std. }}$

\begin{tabular}{|c|c|c|c|c|c|c|c|c|}
\hline \multirow[t]{2}{*}{$\begin{array}{c}\text { Compd. } \\
\text { No. }\end{array}$} & \multicolumn{6}{|c|}{$\begin{array}{c}\text { Antibacterial Activity } \\
(100 \mu \mathrm{g} / \mathrm{ml})\end{array}$} & \multicolumn{2}{|c|}{$\begin{array}{l}\text { Antifungal Activity } \\
(100 \mu \mathrm{g} / \mathrm{ml})\end{array}$} \\
\hline & $\begin{array}{c}\text { B. } \\
\text { subtilis }\end{array}$ & $\begin{array}{c}E . \\
\text { coli }\end{array}$ & $\begin{array}{c}\text { K. } \\
\text { pneumoniae }\end{array}$ & $\begin{array}{c}P . \\
\text { vulgaris }\end{array}$ & $\begin{array}{c}P . \\
\text { auregenosae }\end{array}$ & $\begin{array}{c}\text { S. } \\
\text { typhi }\end{array}$ & $\begin{array}{c}\text { C. } \\
\text { albicans }\end{array}$ & $\begin{array}{c}\text { A. } \\
\text { fumigatus }\end{array}$ \\
\hline $7 \mathbf{a}$ & $\begin{array}{c}12.0 \\
(0.40)^{\mathrm{C}_{1}} \\
(0.42)^{\mathrm{C}}{ }_{2} \\
\end{array}$ & $\begin{array}{c}21.0 \\
(0.75)^{\mathrm{C}_{1}} \\
(0.95)^{\mathrm{C}_{2}}\end{array}$ & $\begin{array}{c}23.0 \\
(1.15)^{\mathrm{C}}{ }_{1} \\
(1.00)^{\mathrm{C}}{ }_{2} \\
\end{array}$ & $\begin{array}{c}20.0 \\
(0.62)^{\mathrm{C}}{ }_{1} \\
(0.66)_{2}^{\mathrm{C}_{2}}\end{array}$ & $\begin{array}{c}22.0 \\
(1.04)^{\mathrm{C}}{ }_{1} \\
(1.10)^{\mathrm{C}}{ }_{2}\end{array}$ & $\begin{array}{c}20.0 \\
(0.51)^{C_{1}} \\
(0.64)_{2}^{C_{2}}\end{array}$ & $\begin{array}{c}26.0 \\
(1.04)^{\mathrm{C}}{ }_{1} \\
(1.36)_{2}^{C_{2}} \\
\end{array}$ & $\begin{array}{c}23.0 \\
(1.00)^{\mathrm{C}}{ }_{1} \\
(0.95)^{\mathrm{C}}{ }_{2} \\
\end{array}$ \\
\hline $7 b$ & $\begin{array}{c}18.5 \\
(0.61)^{C_{1}} \\
(0.66)^{C_{2}}\end{array}$ & $\begin{array}{c}24.7 \\
(0.88)^{C_{1}} \\
(1.12)^{C_{2}}\end{array}$ & $\begin{array}{c}26.0 \\
(1.30)^{\mathrm{C}}{ }_{1} \\
(1.13)^{\mathrm{C}_{2}} \\
\end{array}$ & $\begin{array}{c}22.3 \\
(0.69)^{\mathrm{C}_{1}} \\
(0.74)^{\mathrm{C}}{ }_{2}\end{array}$ & $\begin{array}{c}23.5 \\
(1.11)^{C_{1}} \\
(1.17)^{C_{2}} \\
\end{array}$ & $\begin{array}{c}24.5 \\
(0.62)^{\mathrm{C}}{ }_{1} \\
(0.79)^{\mathrm{C}}{ }_{2}\end{array}$ & $\begin{array}{c}27.8 \\
(1.11)^{C_{1}} \\
(1.46)^{C}{ }_{2} \\
\end{array}$ & $\begin{array}{c}25.0 \\
(1.08)^{\mathrm{C}}{ }_{1} \\
(1.04)_{2}^{\mathrm{C}_{2}}\end{array}$ \\
\hline $7 c$ & $\begin{array}{c}13.2 \\
(0.44)^{C_{1}} \\
(0.47)^{C_{2}}\end{array}$ & $\begin{array}{c}21.0 \\
(0.75)^{C_{1}} \\
(0.95)^{C_{2}}\end{array}$ & $\begin{array}{c}22.9 \\
(1.14)^{\mathrm{C}}{ }_{1} \\
(0.99)^{\mathrm{C}}{ }_{2}\end{array}$ & $\begin{array}{c}18.7 \\
(0.58)^{\mathrm{C}_{1}} \\
(0.62)^{\mathrm{C}}{ }_{2}\end{array}$ & $\begin{array}{c}20.2 \\
(0.96)^{C_{1}} \\
(1.01)^{C_{2}}\end{array}$ & $\begin{array}{c}23.0 \\
(0.58)^{\mathrm{C}_{1}} \\
(0.74)^{\mathrm{C}_{2}}\end{array}$ & $\begin{array}{c}24.1 \\
(0.96)^{C_{1}} \\
(1.26)^{C_{2}}\end{array}$ & $\begin{array}{c}22.0 \\
(0.95)^{\mathrm{C}}{ }_{1} \\
(0.91)_{2}^{\mathrm{C}_{2}}\end{array}$ \\
\hline $7 d$ & $\begin{array}{c}20.9 \\
(0.69)^{\mathrm{C}}{ }_{1} \\
(0 . .74)^{\mathrm{C}}{ }_{2}\end{array}$ & $\begin{array}{c}26.0 \\
(0.92)^{C_{1}} \\
(1.18)^{C_{2}} \\
\end{array}$ & $\begin{array}{c}28.0 \\
(1.40)^{\mathrm{C}}{ }_{1} \\
(1.20)^{\mathrm{C}}{ }_{2} \\
\end{array}$ & $\begin{array}{c}22.8 \\
(0.71)^{\mathrm{C}}{ }_{1} \\
(0.76)_{2}^{\mathrm{C}_{2}}\end{array}$ & $\begin{array}{c}24.6 \\
(1.17)^{\mathrm{C}}{ }_{1} \\
(1.23)^{\mathrm{C}}{ }_{2} \\
\end{array}$ & $\begin{array}{c}26.0 \\
(0.66)^{\mathrm{C}}{ }_{1} \\
(0.83)^{\mathrm{C}_{2}}\end{array}$ & $\begin{array}{c}28.7 \\
(1.14)^{\mathrm{C}}{ }_{1} \\
(1.51)^{\mathrm{C}}{ }_{2} \\
\end{array}$ & $\begin{array}{c}26.2 \\
(1.13)^{\mathrm{C}}{ }_{1} \\
(1.09)^{\mathrm{C}}{ }_{2} \\
\end{array}$ \\
\hline $7 e$ & $\begin{array}{c}22.0 \\
(0.73)^{\mathrm{C}}{ }_{1} \\
(0.78)^{\mathrm{C}}{ }_{2}\end{array}$ & $\begin{array}{c}26.5 \\
(0.94)^{C_{1}} \\
(1.20)^{C_{2}}\end{array}$ & $\begin{array}{c}28.6 \\
(1.43)^{\mathrm{C}}{ }_{1} \\
(1.24)^{\mathrm{C}}{ }_{2}\end{array}$ & $\begin{array}{c}23.0 \\
(0.71)^{\mathrm{C}_{1}} \\
(0.76)_{2}^{\mathrm{C}_{2}}\end{array}$ & $\begin{array}{c}25.0 \\
(1.19)^{\mathrm{C}}{ }_{1} \\
(1.25)^{\mathrm{C}}{ }_{2}\end{array}$ & $\begin{array}{c}28.0 \\
(0.71)^{\mathrm{C}}{ }_{1} \\
(0.90)^{\mathrm{C}}{ }_{2}\end{array}$ & $\begin{array}{c}30.0 \\
(1.20)^{\mathrm{C}}{ }_{1} \\
(1.57)^{\mathrm{C}}{ }_{2}\end{array}$ & $\begin{array}{c}27.0 \\
(1.17)^{\mathrm{C}}{ }_{1} \\
(1.12)^{\mathrm{C}}{ }_{2}\end{array}$ \\
\hline $7 f$ & $\begin{array}{c}13.0 \\
(0.43)^{\mathrm{C}_{1}} \\
(0.46)^{\mathrm{C}}{ }_{2}\end{array}$ & $\begin{array}{c}19.0 \\
(0.67)^{C_{1}} \\
(0.86)^{C_{2}}\end{array}$ & $\begin{array}{c}22.0 \\
(1.10)^{\mathrm{C}}{ }_{1} \\
(0.95)^{\mathrm{C}}{ }_{2}\end{array}$ & $\begin{array}{c}28.0 \\
(0.87)^{\mathrm{C}}{ }_{1} \\
(0.93)_{2}^{\mathrm{C}_{2}}\end{array}$ & $\begin{array}{c}17.0 \\
(0.80)^{\mathrm{C}}{ }_{1} \\
(0.85)^{\mathrm{C}}{ }_{2}\end{array}$ & $\begin{array}{c}21.6 \\
(0.55)^{C_{1}} \\
(0.69)^{C_{2}}\end{array}$ & $\begin{array}{c}18.3 \\
(0.73)^{\mathrm{C}}{ }_{1} \\
(0.96)^{\mathrm{C}}{ }_{2}\end{array}$ & $\begin{array}{c}22.1 \\
(0.96)^{\mathrm{C}}{ }_{1} \\
(0.92)^{\mathrm{C}}{ }_{2}\end{array}$ \\
\hline $7 g$ & $\begin{array}{c}15 \\
(0.50)^{C_{1}} \\
(0.53)^{C_{2}}\end{array}$ & $\begin{array}{c}23.1 \\
(0.82)^{C_{1}} \\
(1.05)^{C_{2}}\end{array}$ & $\begin{array}{c}24.2 \\
(1.21)^{\mathrm{C}}{ }_{1} \\
(1.05)^{\mathrm{C}}{ }_{2}\end{array}$ & $\begin{array}{c}20.0 \\
(0.62)^{C_{1}}{ }_{1} \\
(0.66)_{2}^{C_{2}}\end{array}$ & $\begin{array}{c}21.5 \\
(1.02)^{C_{1}} \\
(1.07)^{C_{2}}\end{array}$ & $\begin{array}{c}24.1 \\
(0.61)^{C_{1}}{ }_{1} \\
(0.77)_{2}^{C_{2}}\end{array}$ & $\begin{array}{c}17.7 \\
(0.70)^{C_{1}} \\
(0.93)^{C_{2}}\end{array}$ & $\begin{array}{c}23.0 \\
(1.00)^{C_{1}} \\
(0.95)^{C_{2}}\end{array}$ \\
\hline $7 \mathrm{~h}$ & $\begin{array}{c}14.4 \\
(0.48)^{C_{1}} \\
(0.51)^{C_{2}}\end{array}$ & $\begin{array}{c}23.2 \\
(0.82)^{\mathrm{C}}{ }_{1} \\
(1.05)^{\mathrm{C}}{ }_{2}\end{array}$ & $\begin{array}{c}24.0 \\
(1.20)^{\mathrm{C}}{ }_{1} \\
(1.04)^{\mathrm{C}}{ }_{2} \\
\end{array}$ & $\begin{array}{c}21.0 \\
(0.65)^{\mathrm{C}}{ }_{1} \\
(0.70)_{2}^{\mathrm{C}}\end{array}$ & $\begin{array}{c}23.0 \\
(1.09)^{\mathrm{C}}{ }_{1} \\
(1.15)^{\mathrm{C}}{ }_{2}\end{array}$ & $\begin{array}{c}22.3 \\
(0.57)_{1}^{\mathrm{C}}{ }_{1} \\
(0.71)^{\mathrm{C}}{ }_{2}\end{array}$ & $\begin{array}{c}25.0 \\
(1.00)^{\mathrm{C}}{ }_{1} \\
(1.31)^{\mathrm{C}}{ }_{2}\end{array}$ & $\begin{array}{c}23.9 \\
(1.03)^{\mathrm{C}}{ }_{1} \\
(0.99)^{\mathrm{C}}{ }_{2}\end{array}$ \\
\hline $9 a$ & $\begin{array}{c}21.0 \\
(0.70)^{C_{1}} \\
(0.75)^{C_{2}} \\
\end{array}$ & $\begin{array}{c}19.2 \\
(0.68)^{\mathrm{C}}{ }_{1} \\
(0.87)^{\mathrm{C}}{ }_{2}\end{array}$ & $\begin{array}{c}25.1 \\
(1.25)^{\mathrm{C}}{ }_{1} \\
(1.09)^{\mathrm{C}}{ }_{2}\end{array}$ & $\begin{array}{c}20.8 \\
(0.65)^{\mathrm{C}}{ }_{1} \\
(0.69)^{\mathrm{C}}{ }_{2}\end{array}$ & $\begin{array}{c}18.0 \\
(0.85)^{\mathrm{C}}{ }_{1} \\
(0.90)^{\mathrm{C}}{ }_{2}\end{array}$ & $\begin{array}{c}27.0 \\
(0.69)^{\mathrm{C}}{ }_{1} \\
(0.87)^{\mathrm{C}}{ }_{2}\end{array}$ & $\begin{array}{c}22.2 \\
(0.88)^{\mathrm{C}}{ }_{1} \\
(1.16)^{\mathrm{C}}{ }_{2}\end{array}$ & $\begin{array}{c}23.0 \\
(1.00)^{C_{1}} \\
(0.95)^{C_{2}}\end{array}$ \\
\hline $9 b$ & $\begin{array}{c}19.3 \\
(0.64)^{C_{1}} \\
(0.68)^{C_{2}}\end{array}$ & $\begin{array}{c}16.0 \\
(0.57)^{C_{1}} \\
(0.72)^{C_{2}}\end{array}$ & $\begin{array}{c}23.0 \\
(1.15)^{\mathrm{C}}{ }_{1} \\
(1.00)^{\mathrm{C}}{ }_{2}\end{array}$ & $\begin{array}{c}20.0 \\
(0.62)^{C_{1}}{ }_{1} \\
(0.66)_{2}^{C_{2}}\end{array}$ & $\begin{array}{c}16.0 \\
(0.76)^{C_{1}} \\
(0.80)^{C_{2}}\end{array}$ & $\begin{array}{c}25.0 \\
(0.64)^{C_{1}} \\
(0.80)^{C_{2}}\end{array}$ & $\begin{array}{c}24.6 \\
(0.98)^{\mathrm{C}}{ }_{1} \\
(1.29)^{\mathrm{C}}{ }_{2}\end{array}$ & $\begin{array}{c}22.0 \\
(0.95)^{\mathrm{C}}{ }_{1} \\
(0.91)^{\mathrm{C}_{2}}\end{array}$ \\
\hline $9 c$ & $\begin{array}{c}17.0 \\
(0.56)^{C_{1}} \\
(0.60)^{C_{2}}\end{array}$ & $\begin{array}{c}15.7 \\
(0.56)^{C_{1}} \\
(0.71)^{C_{2}}\end{array}$ & $\begin{array}{c}20.0 \\
(1.00)^{\mathrm{C}}{ }_{1} \\
(0.86)^{\mathrm{C}}{ }_{2}\end{array}$ & $\begin{array}{c}18.4 \\
(0.57)^{\mathrm{C}_{1}} \\
(0.61)^{\mathrm{C}_{2}}\end{array}$ & $\begin{array}{c}13.0 \\
(0.61)^{C_{1}} \\
(0.65)^{C_{2}}\end{array}$ & $\begin{array}{c}22.0 \\
(0.56)^{C_{1}} \\
(0.70)^{C_{2}}\end{array}$ & $\begin{array}{c}20.9 \\
(0.83)^{\mathrm{C}}{ }_{1} \\
(1.10)^{\mathrm{C}}{ }_{2}\end{array}$ & $\begin{array}{c}19.0 \\
(0.82)^{C_{1}} \\
(0.79)^{C_{2}}\end{array}$ \\
\hline $\mathrm{C}_{1}$ & 30.0 & 28.0 & 20.0 & 32.0 & 21.0 & 39.0 & 25.0 & 23.0 \\
\hline $\mathrm{C}_{2}$ & 28.0 & 22.0 & 23.0 & 30.0 & 20.0 & 31.0 & 19.0 & 24.0 \\
\hline
\end{tabular}

(Activity index $)=$ Inhibition zone of the sample/Inhibition zone of the standard.

For antibacterial activity: $\mathrm{C}_{1}=$ Ciprofloxacin $\& \mathrm{C}_{2}=$ Gentamicin

For antifungal activity: $\mathrm{C}_{1}=$ Griseofulvin $\& \mathrm{C}_{2}=$ Gentamicin 
The cup or well method developed by Collee, Fraser, Marmion and Simmons ${ }^{25}$ has been followed. The test organisms used for antibacterial studies were Escherichia coli, Proteus vulgaris, Klebsiella pneumoniae, Pseudomonas auregenosa, Salmonella typhi and Bacillus subtilis. For antifungal activity: $\mathrm{C}_{1}=$ Griseofulvin \& $\mathrm{C}_{2}=$ Gentamicinhe antibacterial investigations, all synthesized compounds have shown very little activity against B.subtilus, $P$. vulgaris and $S$. typhi, moderate activity against $E$. coli and very strong activity against $K$. pneumoniae and $P$. auregenosa as compared to standards used i.e. Ciprofloxacin $\left(\mathrm{C}_{1}\right)$ and Gentamicin $\left(\mathrm{C}_{2}\right)$. Comparative study of the substitution pattern of the aryl group towards antibacterial activity has shown that electron withdrawing and donating group causes, respectively, more and less activity. Compounds $\mathbf{7 d}$ and $\mathbf{7 e}$ in which a nitro group is present at the meta and para position of the aryl ring, respectively, possess stronger antibacterial activity than others. The chlorine atom in $\mathbf{7 b}$, being a less electron withdrawing moiety, shows less activity than $\mathbf{7 d}$ and $\mathbf{7 e}$ and compound $\mathbf{7 a}, \mathbf{7 c}$ and $\mathbf{7 f}$ manifested very little activity compared to $7 \mathrm{~g}$ with a phenyl group because of the electron donating groups i.e. $\mathrm{OCH}_{3}$ and $\mathrm{NMe}_{2}$. Thiazolidinone protected isoniazid with $\omega$-ethoxyphthalimide 9a showed good antibacterial activity compared to $\mathbf{9 b}$ and $\mathbf{9 c}$ containing $\omega$-propoxy and butoxyphthalimide respectively. 7a-h and 9a-c were also evaluated for antifungal activity against $A$. fumigatus and $C$. albicans. In case of $A$. fumigatus compounds $\mathbf{7 b}, \mathbf{7 d}$ and $\mathbf{7 e}$ showed a better activity than standards i.e. Griseofulvin $\left(\mathrm{C}_{1}\right)$ and gentamicine $\left(\mathrm{C}_{2}\right)$. Compounds $\mathbf{7 a}, \mathbf{7} \mathbf{c}$ and $\mathbf{7 h}$ exhibited comparable activity to standard drugs and remaining compounds $7 \mathbf{f}, \mathbf{7 g}$ and $9 \mathbf{a}-\mathbf{c}$ exhibited moderate activities as compared to the standards. Up to some extent, the effect of the substitution pattern of the aryl group has been similar to antibacterial investigations. In conclusion, the results of the antimicrobial screening reveal that some of the synthesized compounds viz. 7b, 7d, 7e and 9a exhibit good antibacterial and antifungal activity and they can be developed as potent chemotherapeutic agents.

\section{Experimental Section}

General Procedures. Melting points of all synthesized compounds were determined in open capillary tubes and are uncorrected. IR spectra $(\mathrm{KBr})$ and ${ }^{1} \mathrm{H}$ NMR spectra $\left(\mathrm{DMSO}-\mathrm{d}_{6}\right)$ were recorded on FTIR RXI Perkin-Elmer 1800 spectrophotometer and DRX-300 (300 MHz) spectrophotometer using TMS as internal standard, respectively and mass spectra were recorded on a Jeol SX-102 (FAB) spectrometer. The purity of compounds was checked by elemental analysis and also by TLC using silica gel "G", as adsorbent and visualization was accomplished by Iodine.

Isonicotinoylthiosemicarbazide (2). Isoniazid ( 0.01 mole) was dissolved in a minimum amount of $1 \mathrm{~N} \mathrm{HCl}$ and ammonium thiocyanate $(0.02 \mathrm{~mole})$ was added afterward. The reaction mixture was heated under reflux for 8-10 hr. After cooling, the product was filtered, washed with water and recrystallized from absolute alcohol. 2 : Yield $67 \%$, m.p. $207^{\circ} \mathrm{C}$; IR $(\mathrm{KBr}) \mathrm{cm}^{-1}: 3315$ \& 
3178 (N-H str.), 3081-3011 (C-H, Ar-H), 1644-1430 (C=N), $691(\mathrm{C}=\mathrm{S}) ;{ }^{1} \mathrm{H}$ NMR (DMSO-d 6 ): $\delta$ $8.43(\mathrm{~d}, 2 \mathrm{H}, \mathrm{Ar}-\mathrm{H}$ proton near $\mathrm{N}$ in pyridine ring), 8.11 (s, 1H, CONH), 6.77 (d, 2H, Ar-H proton of pyridine ring), $5.1\left(\mathrm{~s}, 2 \mathrm{H}, \mathrm{NH}_{2}\right.$ ); Anal. Calcd. for $\mathrm{C}_{7} \mathrm{H}_{8} \mathrm{~N}_{4} \mathrm{OS}: \mathrm{C}, 42.86 ; \mathrm{H}, 4.08 ; \mathrm{N}, 28.57$. Found: C, 42.82; H, 4.00; N, 28.55\%.

2-Amino-5-(4'-pyridyl)-1,3,4-thiadiazole (3). Isonicotinoylthiosemicarbezide 2 (0.01 mole) was dissolved in $4 \mathrm{~mL}$ of conc. sulphuric acid. Then, the solution was kept at room temperature for $2 \mathrm{hr}$, stirred occasionally and then poured over crushed ice. The resulting solid was kept in ammonical water for $2 \mathrm{hr}$ and was then filtered, washed with water and recrystallized from ethanol as light brown colored crystals. 3 : Yield 70\%, m.p. $240^{\circ} \mathrm{C}$; IR $(\mathrm{KBr}) \mathrm{cm}^{-1}: 3340(\mathrm{~N}-\mathrm{H}$ str.), 1621-1433 (C=N), $660(\mathrm{C}-\mathrm{S}-\mathrm{C}) ;{ }^{1} \mathrm{H}$ NMR (DMSO-d $): \delta 8.50(\mathrm{~d}, 2 \mathrm{H}$, Ar-H, proton of pyridine ring), 6.51 (s, 2H, $\mathrm{NH}_{2}$ ); Anal. Calcd. for $\mathrm{C}_{7} \mathrm{H}_{6} \mathrm{~N}_{4} \mathrm{~S}: \mathrm{C}, 47.19 ; \mathrm{H}, 3.37$; N, 31.46. Found: C, 47.12; H, 3.28; N, 31.21\%.

2-[(4-Methoxyphenyl)methylene]-5-(4'-pyridyl)-1,3,4-thiadiazole (4a). A mixture of 3 (0.01 mole) and anisaldehyde (0.01 mole) was refluxed in ethanol for 6-7 hr with a few drops of glacial acetic acid. The solid separated on cooling was filtered, dried and recrystallized from benzene as needle shaped cream colored crystals. 4a : Yield 74.5\%, m.p. $169^{\circ} \mathrm{C}$; IR $(\mathrm{KBr}) \mathrm{cm}^{-1}$ : 3033 (Ar-H), 2928 (C-H str., $\left.\mathrm{CH}_{3}\right) 1666$ (C=N, exocyclic), 1610 (C=N, cyclic), 690 (C-S); ${ }^{1} \mathrm{H}$ NMR (DMSO-d $): \delta 7.71(\mathrm{~s}, 1 \mathrm{H}, \mathrm{N}=\mathrm{CH}), 7.07$ (d, 2H, Ar-H), $6.83(\mathrm{~d}, 2 \mathrm{H}, \mathrm{Ar}-\mathrm{H}), 6.80(\mathrm{~d}$, $2 \mathrm{H}, \mathrm{Ar}-\mathrm{H}$, near $\left.\mathrm{OCH}_{3}\right), 3.42\left(\mathrm{~s}, 3 \mathrm{H}, \mathrm{OCH}_{3}\right)$; Anal. Calcd. for $\mathrm{C}_{15} \mathrm{H}_{12} \mathrm{~N}_{4} \mathrm{OS}$ : C, 60.81; H, 4.05; N, 18.91. Found: C, 60.77; H, 4.01; N, 18.86\%.

Compounds $\mathbf{4 b}$-h were also prepared in a similar way with minor modifications in reflux time. Their characteristic spectral data are given below:

2-[(4-Chlorophenyl)methylene]-5-(4'-pyridyl)-1,3,4-thiadiazole (4b). Yield 73\%, m.p. $155^{\circ} \mathrm{C}$; IR (KBr) cm $\mathrm{cm}^{-1}$ : 3081-3011 (C-H str, Ar-H), 1663 (C=N exocyclic), 1610-1491 (C=C), $755(\mathrm{C}-\mathrm{Cl}), 669(\mathrm{C}-\mathrm{S}-\mathrm{C}) ;{ }^{1} \mathrm{H}$ NMR $\left(\mathrm{DMSO}_{6}\right)$ ) $\delta 8.51$ (d, $2 \mathrm{H}$, Ar-H proton of pyridine ring), $7.21(\mathrm{~d}, 2 \mathrm{H}, \mathrm{Ar}-\mathrm{H}$ proton near $\mathrm{Cl}), 7.14(\mathrm{~s}, 1 \mathrm{H}, \mathrm{N}=\mathrm{CH}), 6.95(\mathrm{~d}, 2 \mathrm{H}$, Ar-H proton of pyridine ring), 6.52 (d, 2H, Ar-H); Anal. Calcd. for $\mathrm{C}_{14} \mathrm{H}_{9} \mathrm{~N}_{4} \mathrm{SCl}$ : C, 55.91; H, 2.99; N, 18.63. Found: C, 55.88; H, 2.93; N, 18.61\%.

2-[(3,4,5-Trimethoxyphenyl)methylene]-5-(4'-pyridyl)-1,3,4-thiadiazole (4c). Yield 67\%, m.p. $167^{\circ} \mathrm{C}$; IR (KBr) cm ${ }^{-1}$ : $2951\left(\mathrm{C}-\mathrm{H}, \mathrm{CH}_{3}\right), 1681$ (C=N exocyclic), 1041 (C-O-C), 655 (C-S$\mathrm{C}) ;{ }^{1} \mathrm{H}$ NMR $\left(\mathrm{DMSO}_{\mathrm{d}}\right): \delta 7.23(\mathrm{~s}, 1 \mathrm{H}, \mathrm{N}=\mathrm{CH}), 6.88\left(\mathrm{~d}, 2 \mathrm{H}, \mathrm{Ar}-\mathrm{H}\right.$ proton near $\left.\mathrm{OCH}_{3}\right), 3.23(\mathrm{~s}$, $\left.9 \mathrm{H}, \mathrm{OCH}_{3}\right)$; Anal. Calcd. for $\mathrm{C}_{17} \mathrm{H}_{16} \mathrm{~N}_{4} \mathrm{O}_{3} \mathrm{~S}$ : C, 57.30; H, 4.49; N, 15.73. Found: C, 57.28; H, $4.43 ; \mathrm{N}, 15.70 \%$.

2-[(3-Nitrophenyl)methylene]-5-(4'-pyridyl)-1,3,4-thiadiazole (4d). Yield 70\%, m.p. $141^{\circ} \mathrm{C}$; IR (KBr) cm $\mathrm{cm}^{-1}$ : 3021 (C-H str, Ar-H), 1677 (C=N exocyclic), 1521 ( $\mathrm{NO}_{2}$ asym. str.), 1333 ( $\mathrm{NO}_{2}$ symm. str.), 855 (C-N), 651 (C-S-C); ${ }^{1} \mathrm{H}$ NMR (DMSO-d $)$ ): $\delta$ 7.73-7.21 (m, 4H, Ar$\mathrm{H}), 7.11(\mathrm{~s}, 1 \mathrm{H}, \mathrm{N}=\mathrm{CH})$; Anal. Calcd. for $\mathrm{C}_{14} \mathrm{H}_{9} \mathrm{~N}_{5} \mathrm{O}_{2} \mathrm{~S}: \mathrm{C}, 54.02$; H, 2.89; N, 22.50. Found: $\mathrm{C}$, $54.00 ; \mathrm{H}, 2.79 ; \mathrm{N}, 22.41 \%$.

2-[(4-Nitrophenyl)methylene]-5-(4'-pyridyl)-1,3,4-thiadiazole $\quad$ (4e). Yield $65 \%$ m.p. $110^{\circ} \mathrm{C}$; IR (KBr) cm ${ }^{-1}$ : 3081 (C-H str, Ar-H), 1682 (C=N exocyclic), 1527 ( $\mathrm{NO}_{2}$ asym. str.), $1332\left(\mathrm{NO}_{2}\right.$ symm. str.); ${ }^{1} \mathrm{H}$ NMR $\left(\mathrm{DMSO}_{-} \mathrm{d}_{6}\right): \delta 7.91\left(\mathrm{~d}, 2 \mathrm{H}\right.$, Ar-H proton near $\left.\mathrm{NO}_{2}\right), 7.21(\mathrm{~d}$, 
2H, Ar-H), 7.09 (s, $1 \mathrm{H}, \mathrm{N}=\mathrm{CH})$; Anal. Calcd. for $\mathrm{C}_{14} \mathrm{H}_{9} \mathrm{~N}_{5} \mathrm{O}_{2} \mathrm{~S}: \mathrm{C}, 54.02 ; \mathrm{H}, 2.89 ; \mathrm{N}, 22.50$. Found: C, 53.95; H, 2.83; N, 22.45\%.

2-[(4-Dimethylaminophenyl)methylene]-5-(4'-pyridyl)-1,3,4-thiadiazole (4f). Yield 63\%, m.p. $163^{\circ} \mathrm{C}$; IR $(\mathrm{KBr}) \mathrm{cm}^{-1}$ : $2966\left(\mathrm{C}-\mathrm{H}, \mathrm{CH}_{3}\right), 1693\left(\mathrm{C}=\mathrm{N}\right.$, exocyclic), $1021(\mathrm{C}-\mathrm{N})$; ${ }^{1} \mathrm{H}$ NMR $\left(\mathrm{DMSO}_{-} \mathrm{d}_{6}\right): \delta 7.79$ (d, 2H, Ar-H near $\left.\mathrm{NMe}_{2}\right), 7.62(\mathrm{~d}, 2 \mathrm{H}, \mathrm{Ar}-\mathrm{H}), 7.18(\mathrm{~s}, 1 \mathrm{H}, \mathrm{N}=\mathrm{CH})$; Anal. Calcd. for $\mathrm{C}_{16} \mathrm{H}_{15} \mathrm{~N}_{5} \mathrm{~S}$ : C, 62.13; H, 4.85; N, 22.64. Found: C, 62.09; H, 4.81; N, 22.63\%.

2-(Phenylmethylene)-5-(4'-pyridyl)-1,3,4-thiadiazole (4g). Yield 68\%, m.p. $160^{\circ} \mathrm{C}$; IR (KBr) $\mathrm{cm}^{-1}: 3019$ (C-H str, Ar-H), 1648 (C=N exocyclic), 661 (C-S); ${ }^{1} \mathrm{H}$ NMR (DMSO-d 6$): \delta 7.21(\mathrm{~m}$, $5 \mathrm{H}, \mathrm{Ar}-\mathrm{H}), 7.01(\mathrm{~s}, 1 \mathrm{H}, \mathrm{N}=\mathrm{CH})$; Anal. Calcd. for $\mathrm{C}_{14} \mathrm{H}_{10} \mathrm{~N}_{4} \mathrm{~S}$ : C, 63.16; H, 3.75; N, 21.05. Found: C, 63.10; H, 3.67; N, 21.00\%.

2-(2-Furyl)-5-(4'-pyridyl)-1,3,4-thiadiazole (4h). Yield $62 \%$, m.p. $123^{\circ} \mathrm{C}$; IR (KBr) $\mathrm{cm}^{-1}$ : 3077-3004 (C-H, Ar-H), 1655 (C=N, exocyclic), 644 (C-S-C); ${ }^{1} \mathrm{H}$ NMR (DMSO-d $): 7.65$ (d, $1 \mathrm{H}$, Ar-H proton of furyl), 7.21 (d, $2 \mathrm{H}$, Ar-H proton of furyl), 6.38 (dd, 1H, Ar-H of furyl ring), $7.18(\mathrm{~s}, 1 \mathrm{H}, \mathrm{N}=\mathrm{CH})$. Anal. Calcd. for $\mathrm{C}_{12} \mathrm{H}_{8} \mathrm{~N}_{4} \mathrm{OS}$ : C, 56.25; H, 3.13; N, 21.87. Found: C, 56.15; $\mathrm{H}, 3.11 ; \mathrm{N}, 21.84$.

2-[(4-Methoxyphenyl)methylene]-3-(5'-(4''-pyridyl)-1',3',4'-thiadiazol-2'-yl)-4-oxothiazolidin-5-yl ethanoic acid (5a). A mixture of Schiff base 4a (0.01 mole) and mercaptosuccinic acid in THF $(30 \mathrm{~mL})$ containing a pinch of $\mathrm{ZnCl}_{2}$ was refluxed for $10 \mathrm{hr}$. The hot solution was filtered and cooled in an ice bath. The solid obtained was filtered, washed with a $10 \% \mathrm{NaHCO}_{3}$ solution and was recrystallized from alcohol. Yield 77.3\%, m.p. $197^{\circ} \mathrm{C}$; IR $(\mathrm{KBr}) \mathrm{cm}^{-1}:$ 3231-2525 br $(\mathrm{OH}$ of $\mathrm{COOH}), 1710(\mathrm{C}=\mathrm{O}), 1669(\mathrm{C}=\mathrm{O}$, cyclic $), 1603(\mathrm{C}=\mathrm{N}), 695$ (C-S-C); ${ }^{1} \mathrm{H}$ NMR (DMSO-d $)$ ): $\delta 10.57$ (s, 1H, OH), 8.55 (d, 2H, Ar-H near N), 7.15 (d, 2H, Ar$\mathrm{H}), 6.88\left(\mathrm{~d}, 2 \mathrm{H}, \mathrm{Ar}-\mathrm{H}\right.$, near $\left.\mathrm{OCH}_{3}\right), 6.82(\mathrm{~d}, 2 \mathrm{H}, \mathrm{Ar}-\mathrm{H}$ pyridine ring), 4.10 (s, 1H, N-CH-Ar), 3.34 (t, $\left.1 \mathrm{H}, \mathrm{CH}-\mathrm{CH}_{2}-\mathrm{CO}\right), 3.09$ (d, $2 \mathrm{H}, \mathrm{CH}-\mathrm{CH}_{2} \mathrm{CO}$ ); Anal. Calcd. for $\mathrm{C}_{19} \mathrm{H}_{16} \mathrm{~N}_{4} \mathrm{O}_{4} \mathrm{~S}_{2}: \mathrm{C}, 53.27$; H, 3.73; N, 13.08. Found: C, 53.21; H, 3.66; N, 13.01\%.

Similarly, other compounds $\mathbf{5 b}$-h were also synthesized and their characteristic analytical data are given below:

2-[(4-Chlorophenyl)methylene]-3-(5'-(4''-pyridyl)-1',3',4'-thiadiazol-2'-yl)-4-oxo-

thiazolidin-5-yl ethanoic acid (5b). Yield 79 \%, m.p. $221^{\circ} \mathrm{C}^{-\mathbf{1}}$; IR (KBr) cm: 3331-2550br (OH of $\mathrm{COOH}), 1713(\mathrm{C}=\mathrm{O}), 1688(\mathrm{C}=\mathrm{O}$, cyclic), $1601(\mathrm{C}=\mathrm{N}), 843(\mathrm{C}-\mathrm{N}), 748(\mathrm{C}-\mathrm{Cl}), 681(\mathrm{C}-\mathrm{S}-\mathrm{C})$; ${ }^{1} \mathrm{H}$ NMR (DMSO-d $\left.)_{6}\right): \delta 10.21(\mathrm{~s}, 1 \mathrm{H}, \mathrm{OH}), 7.13(\mathrm{~d}, 2 \mathrm{H}, \mathrm{Ar}-\mathrm{H}$ near Cl), 6.44 (d, 2H, Ar-H), 4.06 (s, 1H, N-CH-Ar), 3.32 (t, 1H, CH-CH $-\mathrm{CO}$ ), 3.19 (d, 2H, CH-CH $2-\mathrm{CO}$ ); Anal. Calcd. for $\mathrm{C}_{18} \mathrm{H}_{13} \mathrm{~N}_{4} \mathrm{O}_{3} \mathrm{~S}_{2} \mathrm{Cl}$ : C, 49.94; H, 3.00; N, 12.94. Found: C, 49.81; H, 2.87; N, 12.86\%.

2-[(3,4,5,-Trimethoxyphenyl)methylene $]-3-\left(5^{\prime}\right.$-(4'"-pyridyl)-1',3',4'-thiadiazol-2'-yl)-4-oxothiazolidin-5-yl ethanoic acid (5c). Yield 81\%, m.p. $211^{\circ} \mathrm{C}$; IR $(\mathrm{KBr}) \mathrm{cm}^{-1}: 3305-2782 \mathrm{br}(\mathrm{OH}$ of $\mathrm{COOH}), 2981\left(\mathrm{C}-\mathrm{H}, \mathrm{CH}_{3}\right), 1721(\mathrm{C}=\mathrm{O}), 1659$ (C=O, cyclic), 1044 (C-O-C), 669 (C-S-C); ${ }^{1} \mathrm{H}$ NMR (DMSO-d $)_{6}: \delta 11.01(\mathrm{~s}, 1 \mathrm{H}, \mathrm{OH}), 6.73\left(\mathrm{~d}, 2 \mathrm{H}, \mathrm{Ar}-\mathrm{H}\right.$ near $\left.\mathrm{OCH}_{3}\right), 4.02(\mathrm{~s}, 1 \mathrm{H}, \mathrm{N}-\mathrm{CH}-$ $\mathrm{Ar}$ ), 3.38 (t, 1H, CH-CH $2-\mathrm{CO}), 3.21$ (d, 2H, CH-CH$-\mathrm{CO}), 3.11$ (s, 9H, $\mathrm{OCH}_{3}$ ); Anal. Calcd. for $\mathrm{C}_{21} \mathrm{H}_{20} \mathrm{~N}_{4} \mathrm{O}_{6} \mathrm{~S}_{2}$ : C, 51.63; H, 4.09; N, 11.47. Found: C, 51.58; H, 4.01; N, 11.42\%.

2-[(3-Nitrophenyl)methylene]-3-(5'-(4''-pyridyl)-1',3',4'-thiadiazol-2'-yl)-4-oxo-thiazolidin5-yl ethanoic acid (5d). Yield 75\%, m.p. $183^{\circ} \mathrm{C}$; IR (KBr) $\mathrm{cm}^{-1}: 3332-2750$ br (OH of COOH), 
$1738(\mathrm{C}=\mathrm{O}), 1685$ (C=O, cyclic), 1529 ( $\mathrm{NO}_{2}$ asymm. str.), 1351 ( $\mathrm{NO}_{2}$ symm. str.), 677 (C-S-C); ${ }^{1} \mathrm{H}$ NMR (DMSO-d $\left.)_{6}\right): \delta 10.19(\mathrm{~s}, 1 \mathrm{H}, \mathrm{OH}), 7.63$ (m, 4H, Ar-H), 4.15 (s, 1H, N-CH-Ar), $3.28(\mathrm{t}$, $1 \mathrm{H}, \mathrm{CH}-\mathrm{CH}_{2}-\mathrm{CO}$ ), 3.01 (d, $2 \mathrm{H}, \mathrm{CH}-\mathrm{CH}_{2}-\mathrm{CO}$ ); Anal. Calcd. for $\mathrm{C}_{18} \mathrm{H}_{13} \mathrm{~N}_{5} \mathrm{O}_{5} \mathrm{~S}_{2}$ : C, 48.76; $\mathrm{H}$, $2.93 ;$ N, 15.80. Found: C, 48.74; H, 2.87; N, 15.69\%.

2-[(4-Nitrophenyl)methylene]-3-(5'-(4''-pyridyl)-1',3',4'-thiadiazol-2'-yl)-4-oxo-thiazolidin5-yl ethanoic acid (5e). Yield 72\%, m.p. $172^{\circ} \mathrm{C}$; IR (KBr) $\mathbf{c m}^{-1}$ : 3333-2752br (OH of COOH), $1729(\mathrm{C}=\mathrm{O}), 1677$ ( $\mathrm{C}=\mathrm{O}$, cyclic), 1531 ( $\mathrm{NO}_{2}$ asym. str.), 1349 ( $\mathrm{NO}_{2}$ symm. str.), 675 (C-S-C); ${ }^{1} \mathrm{H}$ NMR (DMSO-d $\left.)_{6}\right): \delta 10.91$ (s, 1H, OH), 7.86 (d, 2H, Ar-H near $\mathrm{NO}_{2}$ ), 6.99 (d, 2H, Ar-H), 4.11 (s, 1H, N-CH-Ar), 3.19 (t, 1H, CH-CH $-\mathrm{CO}$ ), 3.00 (d, 2H, CH-CH $-\mathrm{CO}$ ); Anal. Calcd. for $\mathrm{C}_{18} \mathrm{H}_{13} \mathrm{~N}_{5} \mathrm{O}_{5} \mathrm{~S}_{2}$ : C, 48.76; H, 2.93; N, 15.80. Found: C, 48.66; H, 2.81; N, 15.72\%.

2-[(4-Dimethylaminophenyl)methylene]-3-(5'-(4''-pyridyl)-1',3',4'-thiadiazol-2'-yl)-4-oxothiazolidin-5-yl ethanoic acid (5f). Yield 78\%, m.p. $188^{\circ} \mathrm{C}$; IR (KBr) $\mathbf{c m}^{-1}$ : 3312-2747br $(\mathrm{OH}$ of $\mathrm{COOH}), 2973\left(\mathrm{C}-\mathrm{H}, \mathrm{CH}_{3}\right), 1718(\mathrm{C}=\mathrm{O}), 1631(\mathrm{C}=\mathrm{O}$, cyclic), $892(\mathrm{C}-\mathrm{N}), 628(\mathrm{C}-\mathrm{S}-\mathrm{C})$; ${ }^{1} \mathrm{H}$ NMR (DMSO-d $\left.)_{6}\right): \delta 10.82(\mathrm{~s}, 1 \mathrm{H}, \mathrm{OH}), 6.52\left(\mathrm{~d}, 2 \mathrm{H}, \mathrm{Ar}-\mathrm{H}\right.$ near $\left.\mathrm{NMe}_{2}\right), 6.43(\mathrm{~d}, 2 \mathrm{H}, \mathrm{Ar}-\mathrm{H})$, 4.04 (s, 1H, N-CH-Ar), 3.11 (t, 1H, CH-CH $-\mathrm{CO}$ ), 2.91 (d, 2H, CH-CH $-\mathrm{CO}$ ); Anal. Calcd. for $\mathrm{C}_{20} \mathrm{H}_{19} \mathrm{~N}_{5} \mathrm{O}_{3} \mathrm{~S}_{2}$ : C, 54.42; H, 4.31; N, 15.87. Found: C, 54.41; H, 4.27; N, 15.81\%.

2-(Phenylmethylene)-3-(5'-(4''-pyridyl)-1',3',4'-thiadiazol-2'-yl)-4-oxo-thiazolidin-5-yl ethanoic acid (5g). Yield 81\%, m.p. $174^{\circ} \mathrm{C}$; IR (KBr) cm $\mathrm{cm}^{-1}: 3327-2732 \mathrm{br}(\mathrm{OH}$ of $\mathrm{COOH}), 1721$ $(\mathrm{C}=\mathrm{O}), 1640\left(\mathrm{C}=\mathrm{O}\right.$, cyclic), 635 (C-S-C); ${ }^{1} \mathrm{H}$ NMR (DMSO-d $): \delta 10.32(\mathrm{~s}, 1 \mathrm{H}, \mathrm{OH}), 7.73(\mathrm{~m}$, 5H, Ar-H), 4.09 (s, 1H, N-CH-Ar), 3.2 (t, 1H, CH-CH $2-\mathrm{CO}$ ), 2.95 (d, 2H, CH-CH $-\mathrm{CO}$ ); Anal. Calcd. for $\mathrm{C}_{18} \mathrm{H}_{14} \mathrm{~N}_{4} \mathrm{O}_{3} \mathrm{~S}_{2}$ : C, 54.27; H, 3.52; N, 14.07. Found: C, 54.19; H, 3.45; N, 14.00\%.

2-(2-Furyl)-3-(5'-(4'-pyridyl)-1',3',4'-thiadiazol-2'-yl)-4-oxo-thiazolidin-5-yl ethanoic acid (5h). Yield 68\%, m.p. $156^{\circ} \mathrm{C}$; IR (KBr) cm c $^{-1}$ : 3331-2724br (OH of COOH), 3075 (Ar-H), 1741 $(\mathrm{C}=\mathrm{O}), 1634\left(\mathrm{C}=\mathrm{O}\right.$, cyclic); ${ }^{1} \mathrm{H}$ NMR $\left(\mathrm{DMSO}_{-} \mathrm{d}_{6}\right): \delta 10.12(\mathrm{~s}, 1 \mathrm{H}, \mathrm{OH}), 7.65(\mathrm{~d}, 1 \mathrm{H}, \mathrm{Ar}-\mathrm{H}$ proton of furyl), 7.23 (d, 2H, Ar-H proton of furyl), 6.42 (dd, 1H, Ar-H of furyl ring), 4.10 (s, $1 \mathrm{H}, \mathrm{N}-\mathrm{CH}-\mathrm{Ar}), 3.15$ (t, 1H, CH-CH $2-\mathrm{CO}$ ), 3.05 (d, 2H, CH-CH $-\mathrm{CO}$ ); Anal. Calcd. for $\mathrm{C}_{16} \mathrm{H}_{12} \mathrm{~N}_{4} \mathrm{O}_{4} \mathrm{~S}_{2}$ : C, 49.48; H, 3.09; N, 14.43. Found: C, 49.38; H, 3.02; N, 14.29\%.

\section{2-[(4-Methoxyphenyl)methylene]-3-(5'-(4'-pyridyl)-1',3',4'-thiadiazol-2'-yl)-4-oxo-}

thiazolidin-5-yl ethanoyl chloride (6a). Compound 5a $(0.01$ mole) and thionyl chloride $(0.02$ mole) in toluene were refluxed for $80 \mathrm{~min}$. on a water bath. The excess of solvent was removed by distillation. The acid chloride of $\mathbf{5 a}-\mathbf{h}$, thus separated out was dried and recrystallized from ethanol. 6a : Yield 55.0\%, m.p. $180^{\circ} \mathrm{C}$; IR (KBr) cm ${ }^{-1}: 1775(\mathrm{C}=\mathrm{O}, \mathrm{COCl}), 1691(\mathrm{C}=\mathrm{O}$, cyclic) $1613(\mathrm{C}=\mathrm{N}), 792$ (C-Cl), 693 (C-S-C); ${ }^{1} \mathrm{H}$ NMR (DMSO-d $)$ ) $\delta 7.1$ (d, 2H, Ar-H), 6.89 (d, 2H, $\mathrm{Ar}-\mathrm{H}$, near $\left.\mathrm{OCH}_{3}\right), 3.54$ (s, 3H, $\left.\mathrm{OCH}_{3}\right), 7.29$ (s, 1H, N-CH), 3.29 (t, 1H, CH-CH $\left.-\mathrm{CO}\right), 2.94$ (d, $2 \mathrm{H}, \mathrm{CH}-\mathrm{CH}_{2}-\mathrm{CO}$ ); Anal. Calcd. for $\mathrm{C}_{19} \mathrm{H}_{15} \mathrm{~N}_{4} \mathrm{O}_{3} \mathrm{~S}_{2} \mathrm{Cl}$ : C, 51.06; H, 3.38; N, 12.54. Found: C, $51.00 ; \mathrm{H}, 3.31 ; \mathrm{N}, 12.48 \%$. Other acid chlorides $\mathbf{6 b}$-h were also prepared in a similar manner and were characterized by physical and analytical data.

6b. Yield $43 \%$, m.p. $167^{\circ}$ C. 6c. Yield $40 \%$, m.p. $181^{\circ}$ C. 6d. Yield $57 \%$, m.p. $161^{\circ}$ C. 6e. Yield $44 \%$, m.p. $153^{\circ} \mathrm{C}$. 6f. Yield $59 \%$, m.p. $115^{\circ} \mathrm{C}$. 6g. Yield $51 \%$, m.p. $144^{\circ} \mathrm{C}$. 6h. Yield 60\%, m.p. $125^{\circ} \mathrm{C}$. 
Phthalimido [2-\{(4-methoxyphenyl)methylene $\}-3-\left(5^{\prime}-\left(4^{\prime \prime}\right.\right.$-pyridyl)-1',3',4'-thiadiazol-2'-yl)4-oxo-thiazolidin-5-yl]ethanoate (7a). To stirred solution of compound $\mathbf{6 a}(0.01 \mathrm{~mole})$ and $\mathrm{Et}_{3} \mathrm{~N}(0.01 \mathrm{~mole})$ in DMF, N-hydroxyphthalmide (0.01 mole) was added. The reaction mixture was refluxed for $2 \mathrm{hr}$, then it was cooled and precipitate of triethylamine hydrochloride was filtered off. The filterate was slowly poured onto ice with constant stirring. The solid obtained was recrystallized from methanol. 7a : Yield 55.0\%, m.p. $240^{\circ} \mathrm{C}$; IR $(\mathrm{KBr}) \mathrm{cm}^{-1}: 3051(\mathrm{Ar}-\mathrm{H})$, 2933 (C-H str., $\mathrm{CH}_{3}$ ), 2843 (C-H str., $\left.\mathrm{CH}_{2}\right), 1720$ (C=O, CONH), 1634 (C=O, cyclic), 1615 $(\mathrm{C}=\mathrm{N}), 1292(\mathrm{C}-\mathrm{O}), 1222(\mathrm{C}-\mathrm{N}), 895(\mathrm{~N}-\mathrm{O}), 675(\mathrm{C}-\mathrm{S}) ;{ }^{1} \mathrm{H}$ NMR $\left(\mathrm{DMSO}-\mathrm{d}_{6}\right): \delta 8.60(\mathrm{~d}, 2 \mathrm{H}$, Ar-H, proton of pyridine ring), 7.51 (m, 4H, Ar-H), 7.21 (d, 2H, Ar-H), 6.95 (d, 2H, Ar-H proton of pyridine ring), $6.82\left(\mathrm{~d}, 2 \mathrm{H}, \mathrm{Ar}-\mathrm{H}\right.$, proton near $\left.\mathrm{OCH}_{3}\right), 4.15(\mathrm{~s}, 1 \mathrm{H}, \mathrm{N}-\mathrm{CH}), 3.24(\mathrm{t}, 1 \mathrm{H}, \mathrm{CH}-$ $\mathrm{CH}_{2}-\mathrm{CO}$ ), 2.9 (d, 2H, CH-CH $\left.2-\mathrm{CO}\right)$ ); ${ }^{13} \mathbf{C}$ NMR (DMSO-d $)$ ): $169.3\left(\mathrm{CH}_{2} \mathrm{COO}\right), 168.4(\mathrm{CO}$, cyclic), $150.2\left(\mathrm{C}\right.$ near $\mathrm{N}$ of pyridine ring), $134.8(\mathrm{C}=\mathrm{N}), 45.7\left(\mathrm{OCH}_{3}\right), 40.3(\mathrm{CH}$ of thiazolidinone ring), $34.3\left(\mathrm{CH}_{2} \mathrm{COO}\right) ; \mathbf{M S}: \mathbf{m} / \mathbf{z}: 573\left[\mathrm{M}^{+}\right]$, 466, 204, 190, 162, 146, 132, 104, 78; Anal. Calcd. for $\mathrm{C}_{27} \mathrm{H}_{19} \mathrm{~N}_{5} \mathrm{O}_{6} \mathrm{~S}_{2}$ : C, 56.54; H, 3.31; N, 12.21. Found: C, 56.55; H, 3.23; N, $12.17 \%$.

Similarly, other compounds $\mathbf{7 b}$-h were also synthesized and their characteristic analytical data are given below:

Phthalimido [2-\{(4-chlorophenyl)methylene $\}-3-\left(5^{\prime}-\left(4^{\prime \prime}\right.\right.$-pyridyl)-1', 3', $\mathbf{4}^{\prime}$-thiadiazol-2'-yl)-4oxo-thiazolidin-5-yl]ethanoate (7b). Yield 58.0\%, m.p. $211^{\circ} \mathrm{C}$; IR $(\mathrm{KBr}) \mathrm{cm}^{-1}: 1732(\mathrm{C}=\mathrm{O})$, 1630 (CO-N-CO), $1621(\mathrm{C}=\mathrm{N}), 1268$ (C-O), 1238 (C-N), 690 (C-S); ${ }^{1} \mathrm{H}$ NMR (DMSO-d 6 ): $\delta$ 7.59 (m, 4H, Ar-H), 7.37 (d, 2H, Ar-H proton near Cl), 6.92 (d, 2H, Ar-H), 4.29 (s, 1H, N-CH), $3.11\left(\mathrm{~d}, 2 \mathrm{H}, \mathrm{CH}_{2}\right)$; ${ }^{13} \mathrm{C}$ NMR $\left(\mathrm{DMSO}_{-} \mathrm{d}_{6}\right): 171.1\left(\mathrm{CH}_{2} \mathrm{CO}\right), 168.9$ (CO, cyclic), $149.8(\mathrm{C}$ near N of pyridine ring), $134.9(\mathrm{C}-\mathrm{Cl}$, aromatic), $135.5(\mathrm{C}=\mathrm{N}), 40.9(\mathrm{CH}$ of thiazolidinone ring), 35.2 $\left(\mathrm{CH}_{2} \mathrm{COO}\right) ; \mathrm{MS}: \mathbf{m} / \mathbf{z}: 577[\mathrm{M}+2]^{+}$, $575\left[\mathrm{M}^{+}\right]$466, 204, 190, 162, 146, 132, 104, 78; Anal. Calcd. for $\mathrm{C}_{26} \mathrm{H}_{16} \mathrm{~N}_{5} \mathrm{O}_{5} \mathrm{~S}_{2} \mathrm{Cl}$ : C, 54.02; H, 2.77; N, 12.12. Found: C, 53.95; H, 2.71; N, 12.01\%.

Phthalimido [2-\{(3,4,5-trimethoxyphenyl)methylene $\}-3-\left(5^{\prime}-\left(4^{\prime \prime}\right.\right.$-pyridyl)-1', $\mathbf{3}^{\prime}, \mathbf{4}^{\prime}$-thiadiazol2'-yl)-4-oxo-thiazolidin-5-yl]ethanoate (7c). Yield 50.0\%, m.p. 244 ${ }^{\circ}$; IR (KBr) cm ${ }^{-1}: 2934$ $\left(\mathrm{CH}_{3}\right), 1712(\mathrm{C}=\mathrm{O}), 1623(\mathrm{C}=\mathrm{N}), 1240(\mathrm{C}-\mathrm{O}), 1230(\mathrm{C}-\mathrm{N}), 695(\mathrm{C}-\mathrm{S}) ;{ }^{1} \mathrm{H}$ NMR $\left(\mathrm{DMSO}-\mathrm{d}_{6}\right): \delta$ $7.63(\mathrm{~m}, 4 \mathrm{H}, \mathrm{Ar}-\mathrm{H}), 6.78\left(\mathrm{~d}, 2 \mathrm{H}, \mathrm{Ar}-\mathrm{H}\right.$ proton near $\left.\mathrm{OCH}_{3}\right), 4.11(\mathrm{~s}, 1 \mathrm{H}, \mathrm{N}-\mathrm{CH}), 3.26(\mathrm{~s}, 9 \mathrm{H}$, $\left.\mathrm{OCH}_{3}\right), 3.19(\mathrm{t}, 1 \mathrm{H}, \mathrm{CH}), 2.78\left(\mathrm{~d}, 2 \mathrm{H}, \mathrm{CH}_{2}\right) ;{ }^{13} \mathrm{C} \mathrm{NMR}\left(\mathrm{DMSO}-\mathrm{d}_{6}\right): 169.8\left(\mathrm{CH}_{2} \mathrm{COO}\right), 168.1$ (CO, cyclic), 150.1 ( $\mathrm{C}$ near $\mathrm{N}$ of pyridine ring), $149.9\left(\mathbf{C}-\mathrm{OCH}_{3}\right), 135.7(\mathrm{C}=\mathrm{N}), 45.9\left(\mathrm{OCH}_{3}\right)$, 41.3 (CH of thiazolidinone ring), $34.7\left(\mathrm{CH}_{2} \mathrm{COO}\right)$; MS: $\mathbf{~ m} / \mathbf{z}: 633\left[\mathrm{M}^{+}\right]$466, 204, 190, 162, 146, 132, 104, 78; Anal. Calcd. for $\mathrm{C}_{29} \mathrm{H}_{23} \mathrm{~N}_{5} \mathrm{O}_{8} \mathrm{~S}_{2}$ : C, 54.98; H, 3.63; N, 11.05. Found: C, 54.86; H, $3.61 ; \mathrm{N}, 10.93 \%$.

Phthalimido [2-\{(3-nitrophenyl)methylene\}-3-(5'-(4''-pyridyl)-1', 3',4'-thiadiazol-2'-yl)-4oxo-thiazolidin-5-yl]ethanoate (7d). Yield 45\%, m.p. $220^{\circ} \mathrm{C}$; IR (KBr) cm ${ }^{-1}: 1713(\mathrm{C}=\mathrm{O}), 1628$ $(\mathrm{C}=\mathrm{N}), 1510$ ( $\mathrm{NO}_{2}$ asym. str.), 1322 ( $\mathrm{NO}_{2}$ symm. str.), 1232 (C-O), 710 (C-S); ${ }^{1} \mathrm{H}$ NMR $\left(\mathrm{DMSO}_{\mathrm{d}}\right): \delta 8.11(\mathrm{~m}, 4 \mathrm{H}, \mathrm{Ar}-\mathrm{H}), 7.81(\mathrm{~m}, 4 \mathrm{H}, \mathrm{Ar}-\mathrm{H}), 4.41(\mathrm{~s}, 1 \mathrm{H}, \mathrm{N}-\mathrm{CH}), 3.15(\mathrm{~d}, 2 \mathrm{H}$, $\left.\mathrm{CH}_{2} \mathrm{CO}\right) ;{ }^{13} \mathrm{C}$ NMR (DMSO-d 6$)$ : $170.6\left(\mathrm{CH}_{2} \mathrm{COO}\right), 169.3$ (CO, cyclic), 150.3 (C near $\mathrm{N}$ of pyridine ring), $148.1\left(\mathrm{C}-\mathrm{NO}_{2}\right.$, aromatic), $135.2(\mathrm{C}=\mathrm{N}), 41.9(\mathrm{CH}$ of thiazolidinone ring $), 35.9$ 
$\left(\mathrm{CH}_{2} \mathrm{COO}\right)$; MS: $\mathbf{m} / \mathbf{z}: 588\left[\mathrm{M}^{+}\right]$466, 204, 190, 162, 146, 132, 104, 78; Anal. Calcd. for $\mathrm{C}_{26} \mathrm{H}_{16} \mathrm{~N}_{6} \mathrm{O}_{7} \mathrm{~S}_{2}$ : C, 53.06; H, 2.72; N, 14.28. Found: C, 52.99; H, 2.68; N, 14.25\%.

Phthalimido [2-\{(4-nitrophenyl)methylene $\}-3-\left(5^{\prime}-\left(4^{\prime \prime}\right.\right.$-pyridyl)-1',3',4'-thiadiazol-2'-yl)-4oxo-thiazolidin-5-yl]ethanoate (7e). Yield 41\%, m.p. $200^{\circ} \mathrm{C}$; IR $(\mathrm{KBr}) \mathrm{cm}^{-1}: 1725(\mathrm{C}=\mathrm{O}), 1636$

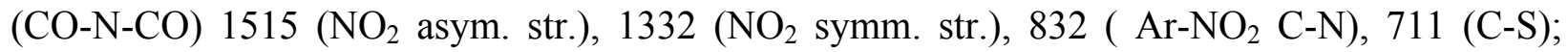
${ }^{1} \mathrm{H}$ NMR (DMSO-d $\left.)_{6}\right): \delta .62\left(\mathrm{~d}, 2 \mathrm{H}, \mathrm{Ar}-\mathrm{H}\right.$ proton near $\left.\mathrm{NO}_{2}\right) 7.43(\mathrm{~d}, 2 \mathrm{H}, \mathrm{Ar}-\mathrm{H}), 7.31(\mathrm{~m}, 2 \mathrm{H}$, Ar-H), 4.28 (s, 1H, N-CH), $3.06\left(\mathrm{t}, 1 \mathrm{H}, \mathrm{CHCH}_{2}\right), 2.61$ (d, $\left.2 \mathrm{H}, \mathrm{CH}_{2} \mathrm{CO}\right) ;{ }^{13} \mathrm{C}$ NMR (DMSO-d 6 ): $170.4\left(\mathrm{CH}_{2} \mathrm{COO}\right), 168.8$ (CO, cyclic), $148.5\left(\mathrm{C}\right.$ near $\mathrm{N}$ of pyridine ring), $148.3\left(\mathbf{C}-\mathrm{NO}_{2}\right.$, aromatic), $136.1(\mathrm{C}=\mathrm{N}), 40.8\left(\mathrm{CH}\right.$ of thiazolidinone ring), $34.9\left(\mathrm{CH}_{2} \mathrm{COO}\right)$; Anal. Calcd. for $\mathrm{C}_{26} \mathrm{H}_{16} \mathrm{~N}_{6} \mathrm{O}_{7} \mathrm{~S}_{2}$ : C, 53.06; H, 2.72; N, 14.28. Found: C, 52.95; H, 2.71; N, 14.20\%.

Phthalimido [2-\{(4-dimethylaminophenyl)methylene\}-3-(5'-(4'"-pyridyl)-1',3',4'-thiadiazol2'-yl)-4-oxo-thiazolidin-5-yl]ethanoate (7f). Yield 43\%, m.p. $235^{\circ} \mathrm{C}$; IR (KBr) cm ${ }^{-1}$ : 3031 (Ar$\mathrm{H}), 1756(\mathrm{C}=\mathrm{O}), 1658$ (CO-N-CO), $1220(\mathrm{C}-\mathrm{N}), 1020$ (C-O), 712 (C-S); ${ }^{1} \mathrm{H}$ NMR (DMSO-d $): \delta$ $7.72(\mathrm{~m}, 4 \mathrm{H}, \mathrm{Ar}-\mathrm{H}), 6.93$ (d, 2H, Ar-H), 6.75 (d, 2H, Ar-H near $\left.\mathrm{NMe}_{2}\right), 3.72\left(\mathrm{~s}, 6 \mathrm{H}, \mathrm{CH}_{3}\right), 3.58$ (d, 2H, $\left.\mathrm{CH}_{2}\right) ;{ }^{13} \mathrm{C}$ NMR (DMSO-d $)$ ) $169.5\left(\mathrm{CH}_{2} \mathrm{COO}\right), 168.2$ (CO, cyclic), 145.9 (C near N of pyridine ring), $136.3(\mathrm{C}=\mathrm{N}), 41.5\left(\mathrm{CH}\right.$ of thiazolidinone ring), $34.8\left(\mathrm{CH}_{2} \mathrm{COO}\right) ; \mathrm{MS}: \mathrm{m} / \mathrm{z}: 586$ $\left[\mathrm{M}^{+} \cdot\right]$ 466, 204, 190, 162, 146, 132, 104; Anal. Calcd. for $\mathrm{C}_{28} \mathrm{H}_{22} \mathrm{~N}_{6} \mathrm{O}_{5} \mathrm{~S}_{2}: \mathrm{C}, 57.33 ; \mathrm{H}, 3.75 ; \mathrm{N}$, 14.33. Found: C, 57.25; H, 3.68; N, 14.21\%.

Phthalimido [2-(phenylmethylene)-3-(5'-(4'"-pyridyl)-1',3', $\mathbf{4}^{\prime}$-thiadiazol-2'-yl)-4-oxothiazolidin-5-yl]ethanoate (7g). Yield 63\%, m.p. 251 ${ }^{\circ} \mathrm{C}$; IR $(\mathrm{KBr}) \mathrm{cm}^{-1}: 1718(\mathrm{C}=\mathrm{O}), 1620$ (CO-N-CO), $1628(\mathrm{C}=\mathrm{N}), 1235(\mathrm{C}-\mathrm{N}), 665$ (C-S); ${ }_{1}^{1} \mathrm{H}$ NMR (DMSO-d 6 ): $\delta 7.93$ (m, 9H, Ar-H), 4.22 (s, 1H, N-CH), 3.31 (t, 1H, $\left.\mathrm{CHCH}_{2} \mathrm{CO}\right), 2.59$ (d, 2H, CHCH $\left.\mathbf{H}_{2} \mathrm{CO}\right) ;{ }^{13} \mathrm{C} \mathrm{NMR}\left(\mathrm{DMSO}-\mathrm{d}_{6}\right)$ : $170.2\left(\mathrm{CH}_{2} \mathrm{COO}\right), 169.1$ (CO, cyclic), 149.3 (C near $\mathrm{N}$ of pyridine ring), $136.7(\mathrm{C}=\mathrm{N}), 41.7(\mathrm{CH}$ of thiazolidinone ring), 35.1 ( $\left.\mathbf{C H}_{2} \mathrm{COO}\right)$; MS: m/z : $543\left[\mathrm{M}^{+}\right]$466, 204, 190, 162, 146, 132, 104; Anal. Calcd. for $\mathrm{C}_{26} \mathrm{H}_{17} \mathrm{~N}_{5} \mathrm{O}_{5} \mathrm{~S}_{2}: \mathrm{C}, 57.46 ; \mathrm{H}, 3.13 ; \mathrm{N}, 12.89$. Found: C, 57.33; H, 3.07; N, $12.82 \%$.

Phthalimido[2-(2-furyl)-3-(5'-(4'-pyridyl)-1',3',4'-thiadiazol-2'-yl)-4-oxo-thiazolidin -5yl]ethanoate (7h). Yield 60\%, m.p. $225^{\circ} \mathrm{C}$; IR (KBr) cm ${ }^{-1}: 1722$ (C=O), 1666 (CO-N-CO), 1268 (C-O), $680(\mathrm{C}-\mathrm{S}) ;{ }^{1} \mathrm{H}$ NMR (DMSO-d $\left.)_{6}\right): \delta 7.76$ (d, 1H, Ar-H proton of furyl), 7.29 (d, 2H, Ar-H proton of furyl), 6.53 (dd, $1 \mathrm{H}$, Ar-H of furyl ring), $3.47\left(\mathrm{~d}, 2 \mathrm{H}, \mathrm{CH}_{2} \mathrm{CO}\right), 2.11\left(\mathrm{~s}, 4 \mathrm{H}, \mathrm{CH}_{2}\right) ;{ }^{13} \mathrm{C}$ NMR (DMSO-d $)_{6}$ : $171.3\left(\mathrm{CH}_{2} \mathrm{COO}\right), 168.4$ (CO, cyclic), 150.2 (C near $\mathrm{N}$ of pyridine ring), 153.3 ( $\mathrm{C}$ near $\mathrm{O}$ in furyl ring), $135.9(\mathrm{C}=\mathrm{N}), 40.7\left(\mathrm{CH}\right.$ of thiazolidinone ring), $35.6\left(\mathrm{CH}_{2} \mathrm{COO}\right)$; MS: m/z : $533\left[\mathrm{M}^{+}\right]$466, 204, 190, 162, 146, 132, 104; Anal. Calcd. for $\mathrm{C}_{24} \mathrm{H}_{15} \mathrm{~N}_{5} \mathrm{O}_{6} \mathrm{~S}_{2}$ : C, 54.03 ; H, 2.81; N, 13.13. Found: C, 54.00; H, 3.01; N, 12.99\%.

2-Isonicotinoylhydrazido-1,3-thiazolidin-4-one (8). Isonicotinoylthiosemicarbazide $\mathbf{2}(0.01$ mole) and chloroacetic acid (0.01 mole) were dissolved in absolute alcohol and then anhydrous sodium acetate $(0.02$ mole) was added to it. The reaction was heated under reflux for $10 \mathrm{hr}$. Excess of solvent was distilled off under reduced pressure and the reaction mixture was then poured onto crushed ice. The precipitate so obtained was filtered, washed with cold water, dried and recrystallized from absolute alcohol. Yield 45\%, m.p. $280^{\circ} \mathrm{C}$; IR $(\mathrm{KBr}) \mathrm{cm}^{-1}: 3344(\mathrm{~N}-\mathrm{H})$, $1711(\mathrm{C}=\mathrm{O}), 1639\left(\mathrm{C}=\mathrm{O}\right.$, cyclic), $1665\left(\mathrm{C}=\mathrm{N}\right.$, exocyclic), $677(\mathrm{C}-\mathrm{S}-\mathrm{C}) ;{ }^{1} \mathrm{H}$ NMR $\left(\mathrm{DMSO}-\mathrm{d}_{6}\right): \delta$ 
8.49 (d, 2H, Ar-H, near N), 8.41 (s, 1H, NH for thiazolidinone ring), 8.21 (s, 1H, CONH); Anal. Calcd. for $\mathrm{C}_{9} \mathrm{H}_{8} \mathrm{~N}_{4} \mathrm{O}_{2} \mathrm{~S}$ : C, 45.76; H, 3.38; N, 23.72. Found: C, 45.69; H, 3.23; N, 23.71\%.

3-N-Ethoxyphthalimido-2-isonicotinoylhydradizo-1,3-thiazolidin-4-one (9a). A mixture of 8 (0.01 mole) and $\omega$-bromoethoxyphthalimide $(0.01$ mole $)$ were dissolved in absolute alcohol. Pyridine (0.02 mole) was added to this reaction mixture as a base. The reaction mixture was refluxed for $6 \mathrm{hr}$. Subsequently, ethanol was distilled off and the crystals were filtered, dried and recrystallized from methanol. Yield 39\%, m.p. $201^{\circ} \mathrm{C}$; IR $(\mathrm{KBr}) \mathrm{cm}^{-1}: 3314(\mathrm{~N}-\mathrm{H}), 3069(\mathrm{Ar}-\mathrm{H})$, $2835\left(\mathrm{C}-\mathrm{H}\right.$ str, $\left.\mathrm{CH}_{2}\right), 1727(\mathrm{C}=\mathrm{O}, \mathrm{CONH}), 1638(\mathrm{C}=\mathrm{O}$ cyclic $), 1462(\mathrm{C}=\mathrm{N}), 1286(\mathrm{C}-\mathrm{N}), 1125$ (C-O), 871 (N-O), 698 (C-S-C); ${ }^{1} \mathrm{H}$ NMR (DMSO-d $)$ ): $\delta 8.54$ (s, 1H, CONH), 8.15 (d, 2H, Ar-H of pyridine), $7.49(\mathrm{~m}, 4 \mathrm{H}, \mathrm{Ar}-\mathrm{H}), 6.73\left(\mathrm{~d}, 2 \mathrm{H}, \mathrm{Ar}-\mathrm{H}\right.$ of pyridine ring), $3.40\left(\mathrm{~s}, 2 \mathrm{H}, \mathrm{CH}_{2}\right), 2.91(\mathrm{t}$, $\left.2 \mathrm{H}, \mathrm{CH}_{2} \mathrm{CH}_{2} \mathrm{O}\right), 2.53\left(\mathrm{t}, 2 \mathrm{H}, \mathrm{CH}_{2}-\mathrm{N}\right) ;{ }^{13} \mathrm{C} \mathrm{NMR}\left(\mathrm{DMSO}-\mathrm{d}_{6}\right): 170.0(\mathrm{CO}-\mathrm{NH}), 169.8(\mathrm{C}=\mathrm{O})$, 149.5 ( $\mathrm{C}$ near $\mathrm{N}$ of pyridine ring), $135.5(\mathrm{C}=\mathrm{N}), 45.1\left(\mathrm{CH}_{2}\right.$ of thiazolidinone ring), $36.7\left(\mathrm{CH}_{2}-\mathrm{O}\right)$, $34.5\left(\mathrm{CH}_{2}-\mathrm{N}\right)$; MS: m/z : $425\left[\mathrm{M}^{+}\right]$190, 162, 146, 132, 121, 106, 104, 78, 76; Anal. Calcd. for $\mathrm{C}_{19} \mathrm{H}_{15} \mathrm{~N}_{5} \mathrm{O}_{5} \mathrm{~S}: \mathrm{C}, 53.64 ; \mathrm{H}, 3.52 ; \mathrm{N}, 16.47$. Found: $\mathrm{C}, 53.61 ; \mathrm{H}, 3.41 ; \mathrm{N}, 16.45 \%$.

Similarly, other compounds $9 \mathrm{~b} \& 9 \mathrm{c}$ were prepared using appropriate $\omega$ bromoalkoxyphthalimide.

3-N-Propoxyphthalimido-2-isonicotinoylhydradizo-1,3-thiazolidin-4-one (9b). Yield 33\%, m.p. $240^{\circ} \mathrm{C}$; IR (KBr) cm ${ }^{-1}$ : $3310(\mathrm{~N}-\mathrm{H}), 3050(\mathrm{Ar}-\mathrm{H}), 2832\left(\mathrm{C}-\mathrm{H}\right.$ str, $\left.\mathrm{CH}_{2}\right), 1731(\mathrm{C}=\mathrm{O}$, CONH), $1679(\mathrm{C}=\mathrm{O}), 1561(\mathrm{C}=\mathrm{N}), 1284(\mathrm{C}-\mathrm{N}), 1190(\mathrm{C}-\mathrm{O}), 870(\mathrm{~N}-\mathrm{O}), 699(\mathrm{C}-\mathrm{S}-\mathrm{C}) ;{ }^{1} \mathrm{H}$ NMR (DMSO-d $\left.)_{6}\right): \delta 8.44(\mathrm{~s}, 1 \mathrm{H}, \mathrm{CONH}), 8.39(\mathrm{~d}, 2 \mathrm{H}, \mathrm{Ar}-\mathrm{H}$ of pyridine $), 7.35(\mathrm{~m}, 4 \mathrm{H}, \mathrm{Ar}-\mathrm{H}), 6.95(\mathrm{~d}$, $2 \mathrm{H}$, Ar-H of pyridine ring), $3.08\left(\mathrm{t}, 2 \mathrm{H}, \mathrm{CH}_{2}-\mathrm{O}\right), 2.88\left(\mathrm{~s}, 2 \mathrm{H}, \mathrm{CH}_{2}\right), 2.72\left(\mathrm{t}, 2 \mathrm{H}, \mathrm{CH}_{2}-\mathrm{N}\right), 2.61$ (quint, $\left.2 \mathrm{H}, \mathrm{CH}_{2} \mathrm{CH}_{2} \mathrm{CH}_{2}\right) ;{ }^{13} \mathrm{C} \mathrm{NMR}\left(\mathrm{DMSO}-\mathrm{d}_{6}\right)$ : $170.4(\mathrm{CO}-\mathrm{NH}), 168.9(\mathrm{C}=\mathrm{O}), 137.9(\mathrm{C}=\mathrm{N})$, $44.2\left(\mathrm{CH}_{2}\right.$ of thiazolidinone ring), $35.8\left(\mathrm{CH}_{2}-\mathrm{O}\right), 34.2\left(\mathrm{CH}_{2}-\mathrm{N}\right) ; \mathrm{MS}: \mathrm{m} / \mathrm{z}: 439\left[\mathrm{M}^{+} \cdot\right], 204,162$, 146, 135, 132, 121, 106, 104, 78; Anal. Calcd. for $\mathrm{C}_{20} \mathrm{H}_{17} \mathrm{~N}_{5} \mathrm{O}_{5} \mathrm{~S}$ : C, 54.66; H, 3.87; N, 15.92. Found: C, 54.62; H, 3.80; N, 15.91\%.

3-N-Butoxyphthalimido-2-isonicotinoylhydradizo-1,3-thiazolidin-4-one (9c). Yield 34\%, m.p. $255^{\circ} \mathrm{C}$; IR (KBr) cm $\mathrm{cm}^{-1}: 3312(\mathrm{~N}-\mathrm{H}), 1719(\mathrm{C}=\mathrm{O}, \mathrm{CONH}), 1633(\mathrm{C}=\mathrm{O}), 1575(\mathrm{C}=\mathrm{N}), 1280$ (C-N), 1195 (C-O), 869 (N-O), 685 (C-S-C); ${ }^{1} \mathrm{H}$ NMR (DMSO-d (D) $\delta 8.55$ (s, 1H, CONH), 8.33 (d, 2H, Ar-H of pyridine), $7.46(\mathrm{~m}, 4 \mathrm{H}, \mathrm{Ar}-\mathrm{H}), 6.89$ (d, 2H, Ar-H of pyridine ring), 3.11 (s, 2H, $\mathrm{CH}_{2}$ ), 2.79 (t, 2H, $\mathrm{CH}_{2}-\mathrm{O}$ ), 2.69 (t, 2H, $\mathrm{CH}_{2}-\mathrm{N}$ ), 2.13 (quint, 4H, $\mathrm{CH}_{2}-\mathrm{CH}_{2}$ ); ${ }^{13} \mathrm{C}$ NMR (DMSO$\left.\mathrm{d}_{6}\right)$ : $169.9(\mathrm{CO}-\mathrm{NH}), 168.7(\mathrm{C}=\mathrm{O}), 136.5(\mathrm{C}=\mathrm{N}), 43.9\left(\mathrm{CH}_{2}\right.$ of thiazolidinone ring $), 35.9\left(\mathrm{CH}_{2^{-}}\right.$ O), $34.9\left(\mathrm{CH}_{2}-\mathrm{N}\right) ; \mathrm{MS}: \mathrm{m} / \mathrm{z}: 453\left[\mathrm{M}^{+}\right], 218,162,146,135,132,121,106,104,78 ;$ Anal. Calcd. for $\mathrm{C}_{21} \mathrm{H}_{19} \mathrm{~N}_{5} \mathrm{O}_{5} \mathrm{~S}: \mathrm{C}, 55.23 ; \mathrm{H}, 4.19 ; \mathrm{N}, 15.45$. Found: $\mathrm{C}, 55.15 ; \mathrm{H}, 4.13 ; \mathrm{N}, 15.39 \%$.

\section{Acknowledgements}

The authors are thankful to the Head, Department of Chemistry, M. L. Sukhadia University, Udaipur (Raj.) for providing laboratory facilities and to the Director, CDRI, Lucknow for providing spectral and analytical data. One of the authors (DPN) is thankful to CSIR for providing essential financial support. 


\section{References}

1. Martin, G.; Lazarus, A. Postgrad Med., 2000, 108, 42.

2. Riley, L. W. Clin. Infect. Dis., 1993, 17 (Suppl.2), S442.

3. World Health Organization, Geneva, WHO Global Tuberculosis Programme, 1997.

4. Hedia, Marrakchi; Gilbert, L.; Annaik, Q. Microbiology, 2000, 146, 289.

5. Darvay, A.; Basarab, T.; McGregor, J. M.; Russell Jones, R. Clin. Exp. Dermatol, 1999, 24, 167.

6. Snider, D. E. Tubercle, 1980, 61, 191.

7. McConnell, R. B.; Cheetham, H. D. Lancent., 1952, 263, 959.

8. Alagarsamy, V.; Venkateshperumal, R.; Sathyabhama, S.; Vaishnavapriya, S.; Sakkarapandi, S.; Revathi, V.; Kalaiselvi, R.; Balamurugan, J.; Sevukarajan, M. Ind. J. Het. Chem., 2002, 11, 327.

9. Panchhamia, V. L.; Parikh, A. R. J. Ind. Chem. Soc., 1989, 66, 250.

10. Lewis, R. The Rise of Antibiotic-Resistant Infections, FDA consumer magazine, Sept. 1955.

11. Cyranski, M. K.; Krygowski, T.M.; Katritzky, A. R.; Schleyer, P. von R. J. Org. Chem., 2002, 67, 1333.

12. Shafiee, A.; Jalilian, A. R.; Rezaei, M. J. Heterocycl. Chem., 2000, 37, 1325.

13. Abramov, M. A.; Dehaen, W.; D’hooge, B.; Petrov, M. L.; Smeet, S.; Toppet, S.; Voets, M. Tetrahedron, 2000, 56, 3933.

14. Zhang, Z.; Yan, J.; Leung, D.; Wang, S.; Costello, P. C.; Sanghera, J.; US6420400, 2002; Chem. Abstr., 2002, 137, 93758p.

15. Schwarz, M.; Page, P.; Pomel, V.; Quattropani, A.; Thomas, R. J. PCT Int Appl. WO 02102799, 2002; Chem. Abstr., 2003, 138, 55968v.

16. Shin, K. J.; Koo, K. D.; Yoo, K. H.; Kang, Y. K.; Park, W. S.; Kim, D. J. Bioorg. Med. Chem. Lett., 2001, 11, 2397.

17. Fujii, K.; Hatano, K.; Yoshida, J. J. Jpn. Kokai. Tokkya Koho JP.; 2001 335570, 2001; Chem. Abstr., 2002, 136, 20074g.

18. Patel, H. D.; Mistry, B. D.; Desai, K. R. Ind. J. Heter. Chem., 2002, 11, 233.

19. Joshi, P. C. Jr.; Joshi, P. C. Sr. J. Ind. Chem. Soc., 1984, 61, 484.

20. Patel, S. V.; Vasavada, J. N.; Joshi, G. B. J. Ind. Chem. Soc., 1984, 61, 560.

21. Troutman, H. D.; Long, L. M. J. Am. Chem. Soc., 1948, 70, 3436.

22. Wolfgang, L. European J. Pharmacol., 1998, 342, 1.

23. Lanan, G. O.; Owoyale, J. A.; Edafiogho, I. O.; Osuide, G. Pharmacy World Journals, 1988, 5 (11), 307.

24. Groutas, W. C.; Brubaker, Stanga, M. A.; Castrisos, J. C.; Crowley, J. P.; Schatz E. T. J. Med. Chem., 1989, 32, 1607.

25. Collee, G. J.; Fraser, G. A.; Marmion, P. B.; Simmons, A. Practical Medical Microbiology, $14^{\text {th }}$ Ed. Vol. 11, Churchill Livingstone, Edinburg, 1996, 163. 